\title{
THREE-DIMENSIONAL GLOBAL MHD SIMULATIONS OF JET FORMATION IN ACTIVE GALACTIC NUCLEI
}

\author{
R. MATSUMOTO
}

Department of Physics, Faculty of Science, Chiba University 1-33 Yayoi-Cho, Inage-ku, Chiba 263, Japan

$<$ matumoto@c.chiba-u.ac.jp>

\section{AND}

\section{K. SHIBATA}

National Astronomical Observatory, Mitaka, Tokyo 181, Japan $<$ shibata@solar.mtk.nao.ac.jp>

Magnetically driven jets from accretion disks are considered to be the most promising models of astrophysical jets. Uchida \& Shibata (1985) and Shibata \& Uchida (1986) first carried out two-dimensional nonlinear MHD simulations of jet formation from a magnetized disk. Matsumoto et al. (1996) applied the Uchida-Shibata model to a gas torus in active galactic nuclei and showed that the surface layer of the torus accretes faster than the equatorial region like an avalanche because magnetic braking most effectively extracts angular momentum from that layer. A magnetized torus subjects to global non-axisymmetric instabilities (Curry \& Pudritz 1996) and local magnetorotational instability (Balbus \& Hawley 1991). We carried out three-dimensional global MHD simulations to show the non-axisymmetric effects on the torus, avalanche flow and jet formation.

We assume that a rotating polytropic torus with constant angular momentum distribution $L=L_{0}$ is imbedded in a spherical, non-rotating isothermal halo. The gravitational field is assumed to be given by a point mass $M$. In a cylindrical coordinate $(r, \varphi, z)$, the dynamical equilibrium of the torus is described by $-G M /\left(r^{2}+z^{2}\right)^{1 / 2}+L_{0}^{2} /\left(2 r^{2}\right)+(n+1) P / \rho=$ const. where $n$ is the polytropic index. We take the radius of the pressure maximum of the torus $\left[r=L_{0}^{2} /(G M)\right]$ as the reference radius $r_{0}$ and take $V_{K 0}=\left(G M / r_{0}\right)^{1 / 2}$ as the unit of velocity. The initial magnetic field is assumed to be uniform and vertical. 

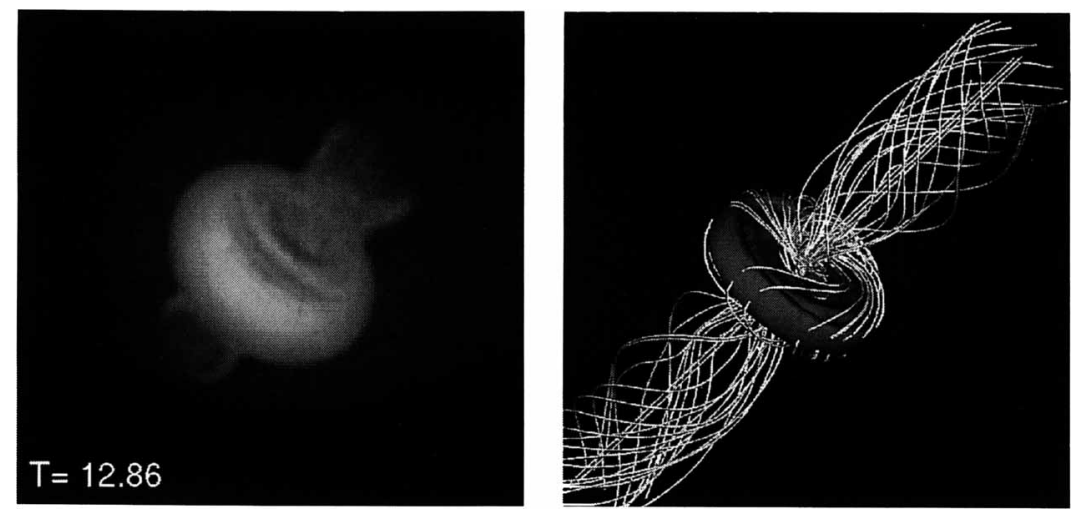

Figure 1. Results of 3D MHD simulation of jet formation. The left panel shows the volume rendered image of density distribution. The right panel shows magnetic field lines and isosurface of density.

In figure 1, we show results of a $3 \mathrm{D}$ MHD simulation of jet formation from a torus. The model parameters are $n=3, \gamma=5 / 3, E_{t h}=$ $\left(a_{0} / V_{K 0}\right)^{2} / \gamma=0.05$, and $E_{m g}=\left(V_{A p 0} / V_{K 0}\right)^{2}=10^{-3}$ where $a_{0}$ and $V_{A p 0}$ are the initial sound speed and Alfvén speed at $(r, z)=\left(r_{0}, 0\right)$, respectively. We initiate the non-axisymmetric evolution by imposing perturbation for azimuthal velocity as $\delta v_{\varphi}=0.01 v_{\varphi} \sin \varphi$. The number of grid points is $\left(n_{r}, n_{\varphi}, n_{z}\right)=(128,32,120)$. We confirmed the results of previous $2 \mathrm{D}$ axisymmetric simulations (Matsumoto et al. 1996) that bipolar jet is formed and that the surface layer of the torus accretes faster than the equatorial part. The avalanche flow creates the radial component of magnetic fields which is further twisted by the differential rotation of the disk. The magnetic field lines at $t=12.86$ indicate that toroidal magnetic field components dominate inside the torus. Due to the growth of non-axisymmetric instabilities, the magnetic field lines are bunched into helical bundles in the jet. A helical filamentary structure can also be seen in the density distribution of the jet. Inside the disk, accretion proceeds along spiral channels. In the innermost region of the disk where toroidal magnetic field becomes dominant, spirally shaped magnetic pressure dominated region appears.

\section{References}

Balbus, S. A., \& Hawley, J. F. 1991, ApJ, 376, 214

Curry, C., \& Pudritz, E. P. 1996, MNRAS, 281, 119

Matsumoto, R., Uchida, Y., Hirose, S., Shibata, K., Hayashi, M. R., Ferrari, A., Bodo, G., \& Norman, C. 1996, ApJ, 461, 115

Shibata, K., \& Uchida, Y. 1986, PASJ, 38, 631

Uchida, Y., \& Shibata, K. 1985, PASJ, 37, 515 